05

\title{
Исследование высокочастотной акустической эмиссии в ходе прерывистой ползучести алюминий-магниевого сплава
}

\author{
(C) А.А. Шибков, М.А. Желтов, М.Ф. Гасанов, А.Е. Золотов, А.А. Денисов, С.С. Кочегаров \\ Тамбовский государственный университет им. Г.Р. Державина, \\ 392000 Тамбов, Россия \\ e-mail: shibkov@tsu.tmb.ru
}

Поступило в Редакцию 21 февраля 2020 г.

В окончательной редакции 17 марта 2020 г.

Принято к публикации 18 марта 2020 г.

Формирование полос макролокализованной деформации в условиях прерывистой ползучести исследовали методами акустической эмиссии (АЭ) и высокоскоростной видеосъемки. Установлено, что наиболее быстрые стадии формирования полосы деформации, связанные с ее выходом на поверхность и последующим ускоренным расширением, сопровождаются генерированием всплеска сигнала АЭ в полосе $\sim 0.05-1 \mathrm{MHz}$. Скрытые корреляции в сложной структуре акустического всплеска исследовали методами статистического и фрактального анализа. Установлена связь между огибающей акустического всплеска и скоростью изменения силового отклика, вызванного формированием одиночной полосы деформации.

Ключевые слова: прерывистая деформация, ползучесть, акустическая эмиссия, полоса деформации, фрактальная структура, алюминий-магниевый сплав.

DOI: 10.21883/JTF.2020.10.49801.61-20

\section{Введение}

Источниками акустической эмиссии (АЭ) в деформируемых кристаллических материалах, как известно, является резкая релаксация внутренних напряжений, связанная с динамикой дислокационных скоплений (линий и полос скольжения), двойников, трещин и т.д., отражающих так называемый мезоскопический структурный уровень пластической деформации кристаллов [1-3]. Несмотря на интенсивные аналитические исследования механизмов генерирования сигналов АЭ на этом уровне [4-7] убедительное экспериментальное доказательство дислокационной природы АЭ получено с помощью кинофильмирования роста двойника в кристалле кальцита и одновременной регистрации акустического сигнала [8]. Исследование методом АЭ пластических неустойчивостей на макроскопическом уровне проводили в основном в условиях проявления эффекта Портевена-Ле Шателье (ПЛШ) - появления повторяющихся скачков напряжения при деформировании с заданной скоростью $\dot{\varepsilon}_{0}=$ const или скачков деформации при нагружении с постоянной скоростью $\dot{\sigma}_{0}=$ const [9-18]. Обнаружено, что каждый скачок напряжения или деформации сопровождается всплеском дискретной АЭ [10], который по визуальным наблюдениям коррелирует с формированием деформационных полос ПЛШ. В работах $[11,12]$ метод АЭ сочетали с лазерной экстенсометрией поверхности деформируемых сплавов системы $\mathrm{Al}-\mathrm{Mg}$. Последняя, однако, имеет недостаточное пространственное разрешение $(\sim 1-2 \mathrm{~mm})$ и быстродействие $(\sim 20 \mathrm{~ms})$ для исследования особенностей динамики деформационных полос ПЛШ различных типов А, В и С. Вместе с тем современные цифровые видеокамеры дают возможность проводить съемку распространяющихся полос локализованной деформации с разрешением $\sim 10 \mu \mathrm{m} /$ pixel и скоростью до нескольких десятков тысяч кадров в секунду [19-23], позволяющей регистрировать наиболее быструю, раннюю стадию формирования полосы, а именно рост зародыша полосы незавершенную полосу, которая пересекает сечение образца за время порядка $1 \mathrm{~ms}$.

В предыдущих работах авторов [22,23] динамика деформационных полос исследовалась методом АЭ в низкочастотной области $\sim 10 \mathrm{~Hz}-10 \mathrm{kHz}$ и синхронно видеосъемкой поверхности с временным разрешением $40-50 \mu \mathrm{s}$ в условиях прерывистой ползучести алюминий-магниевого сплава АМг6. Установлено, что основной вклад в акустический сигнал дает образование завершенной полосы, что подтверждает механизм генерации АЭ дислокациями, выходящими на поверхность образца, а первый пик во всплеске АЭ с временем нарастания $\sim 1 \mathrm{~ms}$ хорошо коррелирует со скачком скорости падения напряжения. Цель настоящей работы - исследовать, какую информацию о динамике индивидуальной полосы деформации несет высокочастотная, в полосе 0.05-1 MHz, дискретная АЭ.

\section{1. Методика}

Материалом исследования является поликристаллический алюминий-магниевый сплав АМг6. Состав сплава, термическая обработка и методика измерения скачков деформации и нагрузки в условиях прерывистой ползучести изложены в работах [22-25]. Отличие настоящей работы состоит в использовании высокочастотного 
датчика AЭ Zetlab ВC601 с почти плоской амплитудночастотной характеристикой (АЧХ) в диапазоне частот $\sim 100-800 \mathrm{kHz}$ и плавным убыванием в области более низких частот, $<100 \mathrm{kHz}$. Сигналы АЭ усиливались на $40 \mathrm{~dB}$ предусилителем AEP5 (Vallen-Systeme) и записывались непрерывно без порога с амплитудным разрешением 16 бит с частотой записи $5 \mathrm{MHz}$. Исследованная ранее в $[22,23]$ низкочастотная область спектра подавлялась фильтром низких частот (до $50 \mathrm{kHz}$ ). Скорость видеосъемки цифровой камерой Fastcam Mini UX 500/100 (Photron) составляла $5000 \mathrm{fps}$ (frames per second). Для контрастирования изображений использовали как и ранее методику вычитаний последовательных цифровых изображений [24].

\section{2. Результаты и обсуждение}

\section{1. Корреляция огибающей сигнала АЭ с кинетикой роста полосы деформации и силовым откликом}

Образцы сплава АМг6 в форме двухсторонних лопаток с размерами рабочей части $6 \times 3 \times 0.25 \mathrm{~mm}$ нагружались в два этапа: сначала с постоянной скоростью роста приложенного напряжения $\dot{\sigma}_{0}=0.1 \mathrm{MPa} / \mathrm{s}$ до фиксированного напряжения ползучести $\sigma_{0}=$ const (270-310 MPa), значительно превышающем условный предел текучести $\sigma_{0.2}=160 \mathrm{MPa}$. Спустя некоторое время (инкубационное время), зависящее от напряжения ползучести, на поверхности образца спонтанно зарождается и растет полоса локализованной пластической деформации, которая является „спусковым крючком“ развития деформационной ступени амплитудой $\sim 1-5 \%$ на кривой ползучести. Обычно полоса зарождается в случайной позиции на поверхности рабочей части образца или вблизи одной из лопаток. Такой случай представлен на рис. 1, который демонстрирует фрагмент записи процесса зарождения и роста незавершенной дугообразной полосы деформации.

Результаты обработки изображений в виде кинетической кривой роста длины $L_{b}$, скорости вершины $v_{t}$ и площади полосы $S$ и ее скорости роста $\bar{S}$, а также акустический сигнал $\varphi_{\mathrm{AE}}(t)$ представлены на рис. 2. Временны́е зависимости геометрических параметров незавершенной полосы демонстрируют типичную нелинейную динамику с резким ускорением на завершающей стадии роста. Отметим, что из-за уменьшения уровня напряжений в области лопатки скорость роста незавершенной дугообразной полосы несколько меньше скорости роста полосы в центральной области рабочей части образца, в которой полоса растет в направлении максимальных касательных напряжений, составляющем угол $58-60^{\circ}$ к оси растяжения [1924]. На момент выхода полосы на противоположную боковую поверхность приходятся пик сигнала АЭ, скорости вершины $v_{t}$ и скорости роста площади поло-

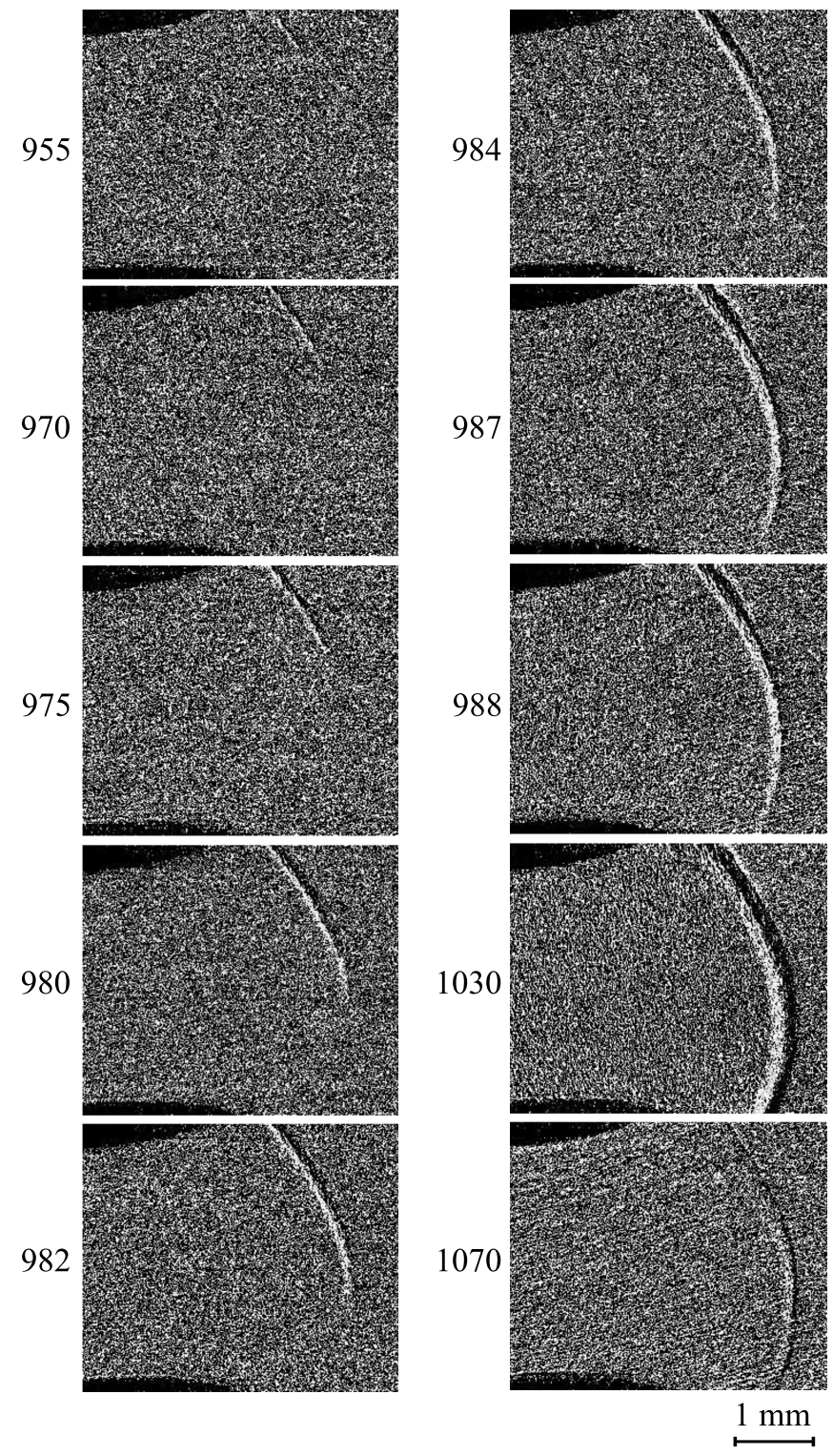

Рис. 1. Формирование первичной полосы деформации, которая является триггером развития скачка деформации в сплаве АМг6 в условиях прерывистой ползучести при $\sigma_{0} \approx 285 \mathrm{MPa}$. Скорость съемки $5000 \mathrm{fps}$. Числа - номера кадров видеосъемки.

сы $\bar{S}$. В отличие от сигнала низкочастотной в полосе $10 \mathrm{~Hz}-10 \mathrm{kHz}$ дискретной АЭ, полученного и исследованного в [22,23], „высокочастотный“ сигнал имеет более сложную структуру в широкой полосе частот (рис. 3) от нижней частоты $50 \mathrm{kHz}$, связанной с фильтрацией низких частот, до $\sim 1 \mathrm{MHz}$, обусловленную в основном верхней частотой АЧХ используемого датчика АЭ.

Таким образом, формирование деформационной полосы сопровождается широкополосным акустическим всплеском, спектр мощности $P(f)$ которого содержит фактически все частоты исследуемого диапазона с основным пиком вблизи $100 \mathrm{kHz}$ (рис. 3) и медианной 


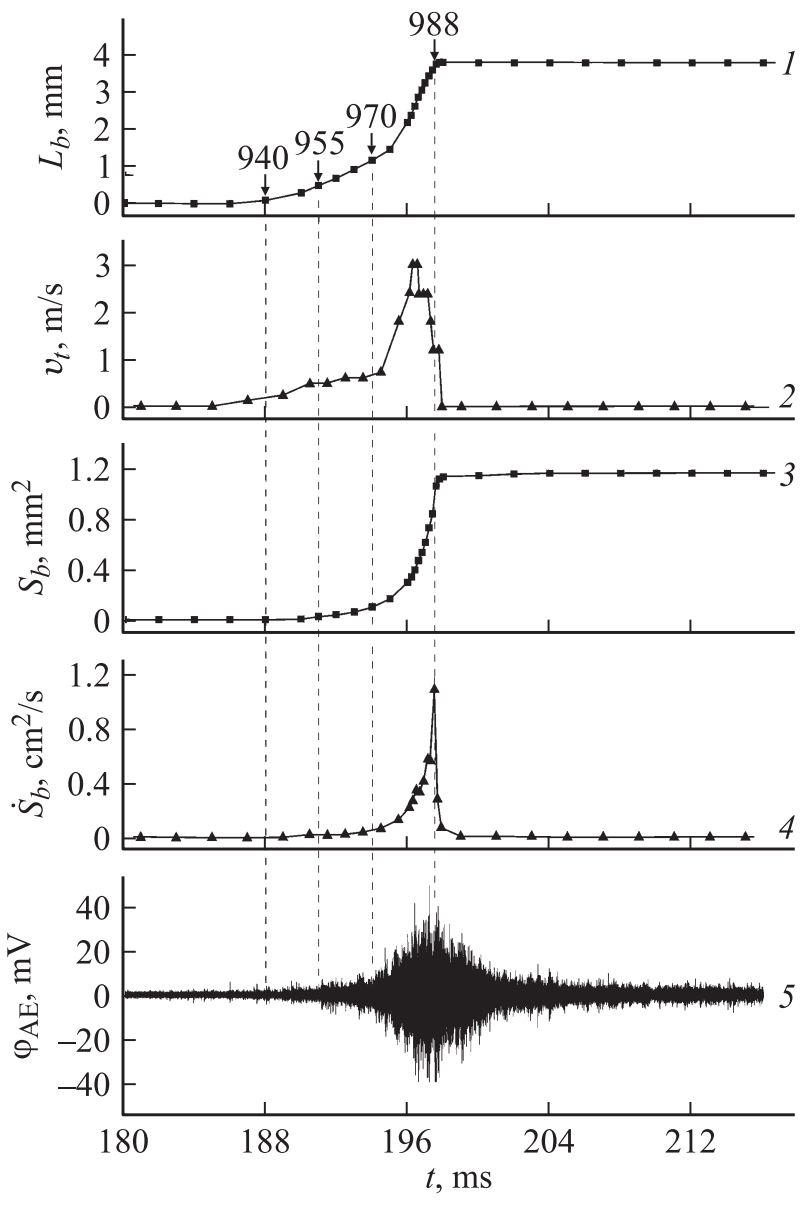

Рис. 2. Временные зависимости различных характеристик первичной полосы деформации: 1 - длина полосы $L_{b}(t)$, 2 - скорость вершины полосы $v_{t}(t), 3$ - площадь полосы $S(t), 4$ - скорость роста площади полосы $\bar{S}(t)$ и 5 - сигнал $\mathrm{A} Э \varphi_{\mathrm{AE}}$.

частотой $f_{m} \approx 300 \mathrm{kHz}$ определяемой, согласно [26], из условия

$$
\int_{0}^{f_{m}} P(f) d f=\int_{f_{m}}^{\infty} P(f) d f,
$$

а огибающая сигнала АЭ имеет колоколообразную форму с максимумом в момент формирования завершенной полосы (рис. 2).

Из рис. 2 следует, что основной вклад в акустический всплеск дает стадия ускоренного роста незавершенной полосы перед выходом ее на противоположную поверхность образца, т.е. моментом образования завершенной полосы. Эта стадия соответствует приблизительно второй половине роста длины полосы (кадры 970-988 на рис. 1). Предшествуюшая стадия со скоростью вершины полосы $v_{t}<0.5 \mathrm{~m} / \mathrm{s}$, включающая собственно момент зарождения полосы, дает относительно небольшой сигнал АЭ, не превышающий уровень непрерывной составляющей акустического сигнала. Стадия затухания акустического всплеска после момента формирования завер- шенной полосы (кадр 988 на рис. 1), приблизительно совпадает со стадией затухания скорости роста площади полосы $\bar{S}_{b}(t)$, длительностью $\sim 1 \mathrm{~ms}$. Соответствующая стадия акустического всплеска предположительно характеризуется динамикой дислокационных лавин внутри полосы, а также возможно эффектами отражения акустических волн от поверхности образца. Последние, однако, возможны только на частотах $f>1 \mathrm{MHz}$, когда длина акустической волны $\lambda$ оказывается сопоставимой или меньше размера образца $d: \lambda=c / f_{\max } \approx 5 \mathrm{~mm}$, где $c \approx 5 \cdot 10^{3} \mathrm{~m} / \mathrm{s}-$ скорость звука. Как видно из рис. 3 , данная область спектра („высокочастотный хвост“) составляет лишь небольшую часть $(<10 \%)$ спектра мощности. Поэтому возможные эффекты отражения акустических волн на частотах $\sim 1 \mathrm{MHz}$ не могут объяснить сложную широкополосную структуру всплеска АЭ, генерируемого в ходе формирования деформационной полосы.

Так как типичная длительность всплеска высокочастотного сигнала АЭ порядка $\sim 3-10 \mathrm{~ms}$, т.е. соизмерима с длительностью фронта скачка напряжения, то представляет интерес исследовать корреляцию осредненной огибающей всплеска АЭ с силовым откликом, точнее с формой функции $\psi(t)=|d \sigma(t) / d t|$. На рис. 4 представлены силовой и акустический отклики на формирование индивидуальной полосы деформации в виде скачка разгрузки $\sigma(t)$ и акустического всплеска $\varphi_{\mathrm{AE}}(t)$ для сравнения с формой функции $\psi(t)$. Осредненная огибающая акустического всплеска рассчитывалась в виде

$$
\xi(t)=\left\langle\left|\varphi_{\mathrm{AE}}(t)\right|\right\rangle=\Delta t^{-1} \int_{t}^{t+\Delta t}\left|\varphi_{\mathrm{AE}}\left(t^{\prime}\right)\right| d t^{\prime},
$$

где $\Delta t$ и $\left|\varphi_{\mathrm{AE}}(t)\right|$ - время осреднения и абсолютное значение сигнала АЭ соответственно. Поскольку нагрузка записывалась со скоростью $2 \mathrm{kHz}$, время осреднения выбиралось равным $\Delta t=0.5 \mathrm{~ms}$. Колоколообразные временные зависимости $\xi(t)$ и $\psi(\xi)$, осредненные на одинаковом времени $\Delta t(=0.5 \mathrm{~ms})$, представлены на рис. 4 (кривые 4 и 5 соответственно). Коэффициент корреляции этих зависимостей, рассчитанный с помощью пакета программ MathCad, $k \approx 0.9316$.

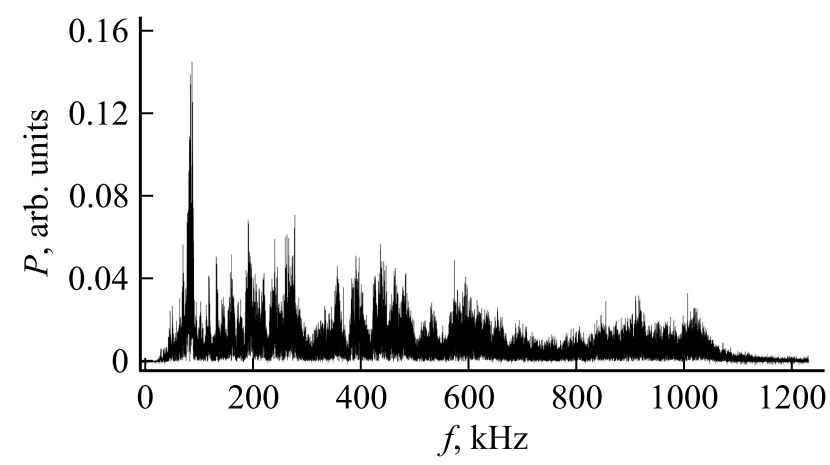

Рис. 3. Спектр мощности $P(f)$ сигнала АЭ, представленного на рис. 2, кривая 5 . 
Другим признаком корреляции функций $\xi(t)$ и $\psi(\xi)$ является приблизительно линейная зависимость между их амплитудами $\Delta \xi_{m}$ и $\Delta \psi_{m}$ (рис. 5). Таким образом, осредненная огибающая модуля высокочастотного акустического сигнала несет информацию о скорости изменения силового отклика $|\dot{\sigma}|$. Этот результат совпадает с выводами работы [23], в которой исследовались корреляции функции $\psi(t)$ с амплитудой низкочастотного (в полосе $10 \mathrm{~Hz}-10 \mathrm{kHz}$ ) сигнала АЭ, вызванного формированием отдельных полос деформации.

Из рис. 5 видно, что акустический всплеск, сопровождающий формирование единичной полосы деформации, имеет сложную фракталоподобную структуру, состоящую из большого количества коротких, длительностью $\sim 1-10 \mu \mathrm{s}$, дискретных импульсов различной амплитуды. Естественно предположить, что эти импульсы АЭ связаны с динамикой дислокационных лавин разного масштаба, участвующих в эволюции сложной пространственно-временной структуры полосы макроло-
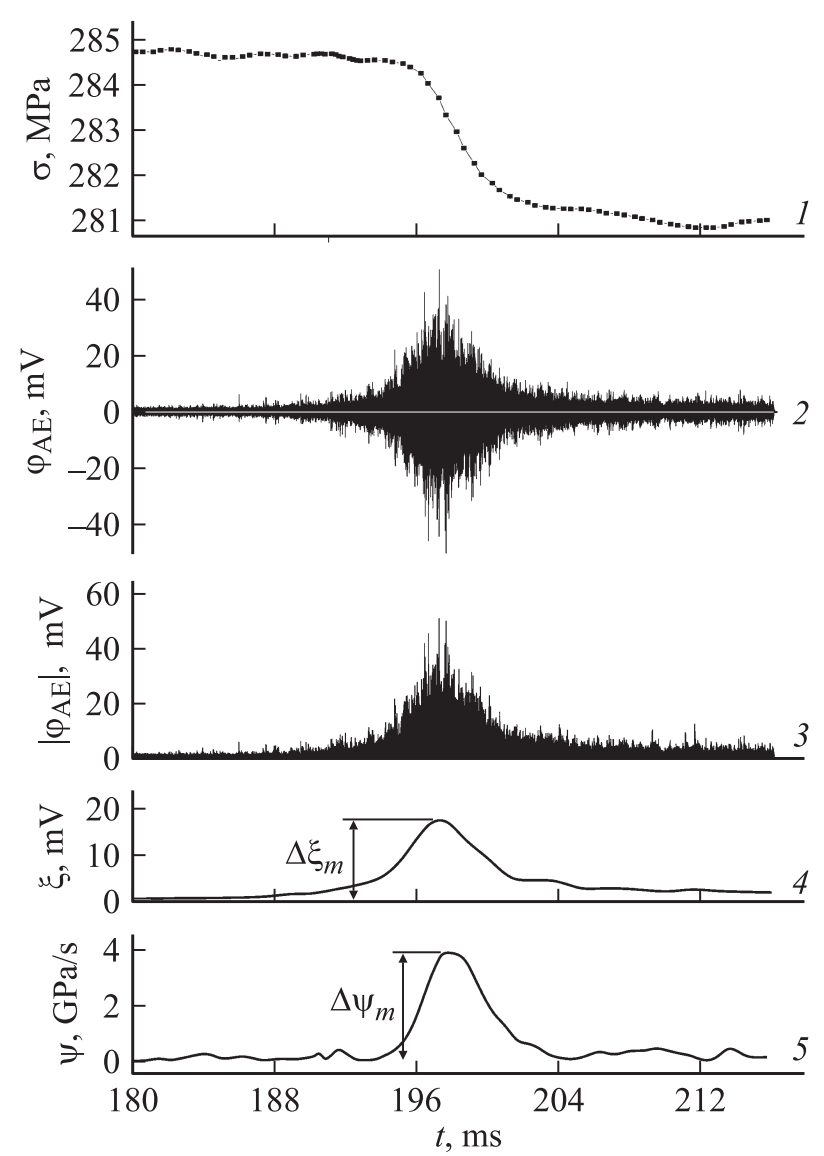

Рис. 4. Скачок разгрузки $\sigma(1)$ и акустический всплеск $\varphi_{\mathrm{AE}}(2)$, вызванный формированием индивидуальной полосы деформации, абсолютная величина сигнала АЭ $\left|\varphi_{\mathrm{AE}}\right|$ (3), его огибающая $\xi(4)$ и абсолютная скорость изменения напряжения $\phi=|\dot{\sigma}|(5) . \Delta \xi_{m}$ и $\Delta \psi_{m}$ - амплитуды огибающей акустического всплеска и скачка скорости изменения напряжения соответственно.

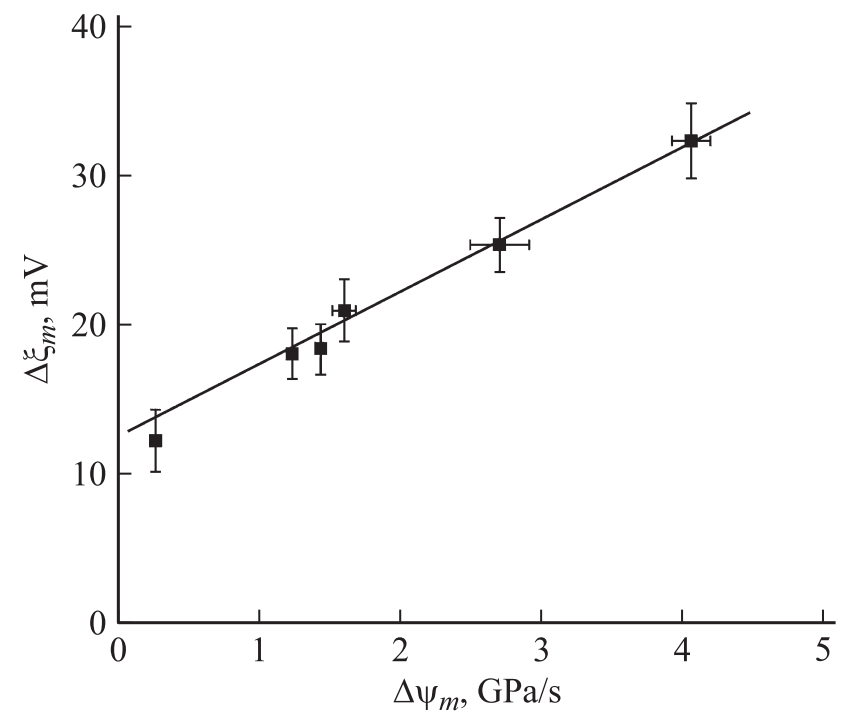

Рис. 5. Зависимость амплитуды огибающей $\Delta \xi_{m}$ всплеска АЭ, вызванного формированием одиночной полосы деформации, от амплитуды скорости изменения силового отклика $\Delta \psi_{m}$.

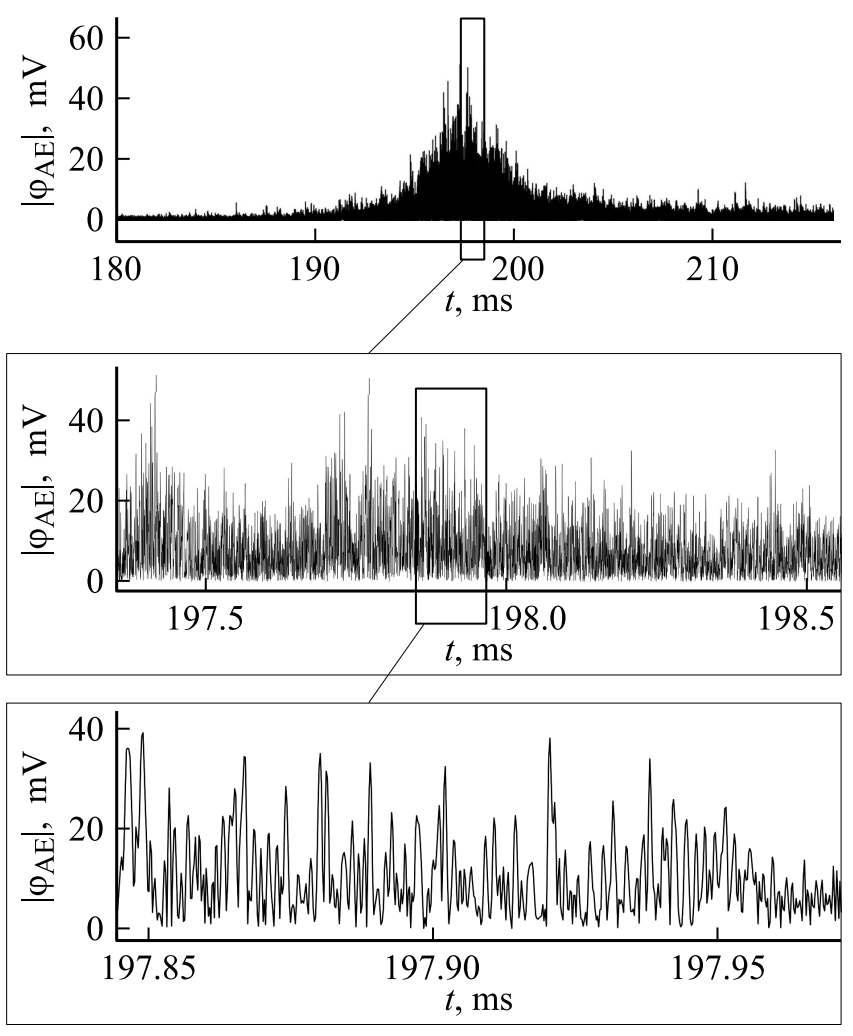

Рис. 6. Временна́я структура при различных временных масштабах всплеска АЭ, вызванного формированием индивидуальной полосы деформации в сплаве АМг6.

кализованной деформации. Поэтому представляет интерес исследовать временну́ю структуру акустического всплеска методами статистического, корреляционного и фрактального анализа. 

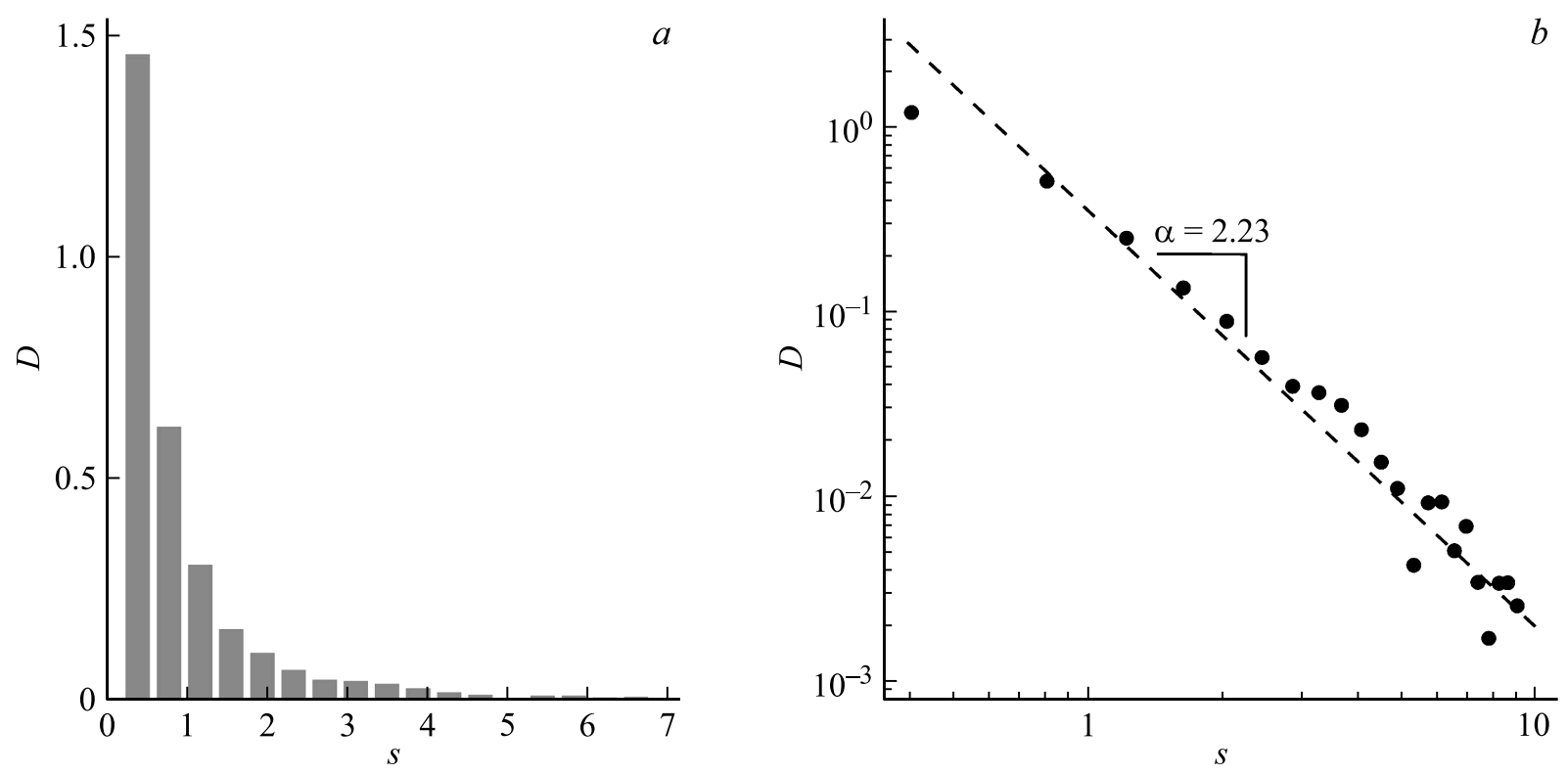

Рис. 7. Амплитудное распределение дискретных сигналов АЭ в структуре акустического всплеска, вызванного формированием отдельной полосы деформации: $a-$ в координатах $D-s, b-$ в двойных логарифмических координатах.

\section{2. Статистический и фрактальный анализ структуры акустического отклика}

Сначала устанавливался нижний порог амплитуды сигнала в структуре акустического всплеска, вдвое превышающем среднеквадратичное значение сигнала АЭ за несколько секунд до момента зарождения первичной полосы деформации (приблизительно в середине плато между ступенями на кривой ползучести). Затем подсчитывалось общее количество $N$ импульсов АЭ над этим порогом (в примере, представленном на рис. 6, $N=2643$ ) и строилась статистическая функция распределения $D(s)=N^{-1} d N / d s$ нормализованной энергии акустических импульсов $s=A^{2} / \bar{A}^{2}$, где $A=\varphi_{m}$ амплитуда импульса АЭ, $A^{2}(=E)$ - энергетическая характеристика единичного импульса АЭ в структуре всплеска АЭ, $\bar{A}^{2}$ - среднее значение квадрата амплитуды сигналов АЭ, $d N-$ количество импульсов АЭ, амплитуда которых попадает в узкий интервал $(s-\delta s / 2, s+\delta s / 2)$.

Статистическая функция распределения квадратов амплитуд $D(s)$, представленная на рис. 7, имеет гиперболическую форму, т. е. сильно отличается от нормального распределения, характерного для случайного процесса. В двойных логарифмических координатах зависимость $\lg D$ от $\lg s$ является приблизительно линейной с коэффициентом наклона к $s-$ оси, равным -2.23 . Это означает, что амплитудное распределение $D(s)$ подчиняется степенному закону

$$
D(s) \sim s^{-\alpha}
$$

с показателем степени $\alpha=2.23$. Степенное распределение квадратов амплитуд импульсов АЭ свидетельствует о самоподобии акустического сигнала, т. е. об отсутствии выделенного масштаба событий (масштабная инвариантность), характерного для фрактальных временных рядов [27,28].

Полученный результат согласуется с результатами численного моделирования самоорганизующихся дислокационных лавин на второй и третьей стадии ползучести [29], а также данными измерения сигналов АЭ при ползучести монокристаллического льда [30,31] и акустических всплесков в условиях проявления эффекта ПЛШ в металлах [32-35]. Численное исследование на монокристаллах льда, для которых характерно скольжение в базисной плоскости, показывает, что в ходе ползучести образуются дислокационные диполи и стенки, которые формируют структуру сил закрепления, что приводит к образованию дислокационных лавин разного масштаба и амплитудное распределение сигналов АЭ демонстрирует степенной закон [29]. Для чистых монокристаллов льда, $\mathrm{Cu}, \mathrm{Zn}$ и $\mathrm{Cd}$ показатель степени $\alpha \approx 1.8-2.15$ [36,37]. Экспериментальные исследования дискретной АЭ в условиях эффекта ПЛШ на поликристаллических сплавах $\mathrm{Al}-\mathrm{Mg}$ с содержанием магния $3-6 \%$ дают $\alpha$ в интервале от 2 до 3 [32-35]. Большие значения $\alpha$ обусловлены, как предполагается в [32], множественным скольжением, наличием преципитатов, границ зерен, дислокаций леса и примесных атомов, как эффективных стопоров движению дислокаций, что увеличивает долю мелких дислокационных лавин и уменьшает долю крупных и, следовательно, увеличивает показатель степени $\alpha$.

Отметим, что классическим примером самоорганизации коллективных динамических процессов является землетрясения. Магнитуда землетрясений измеряется обычно в терминах энергии $E$, высвобождаемой при 


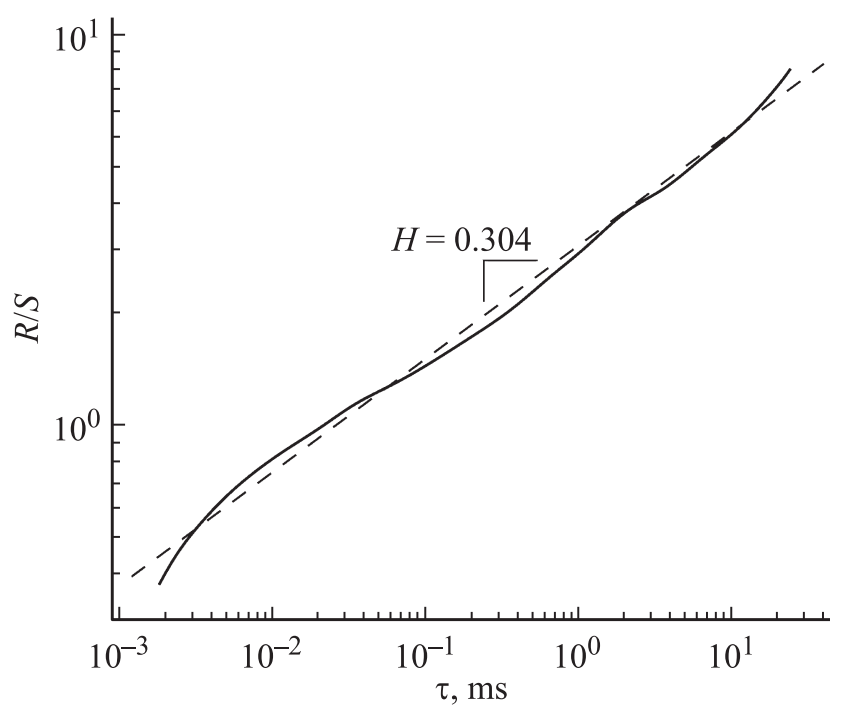

Рис. 8. Результаты $R / S$-анализа Хёрста: зависимость нормированного размаха $R / S$ от $\tau$ в двойных логарифмических координатах; наклон прерывистой линии дает показатель Хёрста $H=0.304$.

скольжении тектонических плит, а статистическое распределение магнитуд $D(E)$ следует степенному закону $D(E) \sim E^{-\alpha}$, где показатель $\alpha$ зависит от географии землетрясения и находится в пределах от 1.8 до 2.2 [27].

Таким образом, спонтанное формирование полос деформации при напряжении ползучести, значительно превышающем предел текучести, по пороговой динамике и статистическому распределению энергии акустических сигналов в структуре всплеска АЭ аналогично землетрясениям и соответствующим сейсмическим сигналам. Они характеризуются медленным накоплением внутренних напряжений и последующей их спонтанной быстрой релаксацией в виде коллективных самоорганизующихся лавин, о чем свидетельствует степенной закон амплитудного распределения сигналов АЭ, который является одним из признаков дальнодействующих корреляций, охватывающих всю систему или значительную ее часть.

Другим признаком самоподобия и наличия скрытых корреляций акустического сигнала является его монофрактальность. Фрактальную размерность сигнала вычисляли, используя $R / S$-анализ Хёрста по методу нормированного размаха [28] с помощью выражения

$$
R / S \sim \tau^{H}
$$

где $R(\tau)=Y_{\max }-Y_{\min }$ - накопленное отклонение (размax) сигнала $\varphi_{\mathrm{AE}}(t)$ от среднего значения на временном интервале $\tau$, а накопленное отклонение определяется как

$$
Y(t, \tau)=\int_{0}^{t}\left[\varphi_{\mathrm{AE}}\left(t^{\prime}\right)-\left\langle\varphi_{\mathrm{AE}}\right\rangle_{\tau}\right] d t^{\prime}
$$

где

$$
\left\langle\varphi_{\mathrm{AE}}\right\rangle_{\tau}=\tau^{-1} \int_{0}^{\tau} \varphi_{\mathrm{AE}}(t) d t
$$

и

$$
S(\tau)=\left\{\tau^{-1} \int_{0}^{\tau}\left[\varphi_{\mathrm{AE}}(t)-\left\langle\varphi_{\mathrm{AE}}\right\rangle_{\tau}\right]^{2} d t^{\prime}\right\}^{1 / 2}
$$

- среднее значение и стандартное отклонение временного ряда $\varphi_{\mathrm{AE}}(t)$ на интервале $\tau, H-$ показатель Хёрста, а фрактальная размерность временного ряда определяется как $D_{f}=2-H[28]$.

На рис. 8 показана зависимость величины $R / S$ от временно́го интервала $\tau$ в двойных логарифмических координатах. Линейная аппроксимация этой зависимости дает значение показателя Хёрста $H=0.304$. Как видно, акустический всплеск, вызванный формированием одиночной полосы деформации, имеет монофрактальную природу с размерностью $D_{f}=1.696$ и скейлингом около полутора порядков по нормированному размаху $R / S$. Отметим, что для белого шума (нескоррелированный процесс) $H=0.5$, в то время как значение $H=0.5$ свидетельствует об антиперсистентности сигнала, состоящей в том, что если амплитуда сигнала возрастает на некотором временном интервале, то следует ожидать, что она будет уменьшаться в следующем временном интервале приблизительно такой же продолжительности, и наоборот, если амплитуда сигнала уменьшается на интервале $\tau$, то в результате внутренней корреляции сигнала его амплитуда будет расти на последующем временном интервале такой же длительности.

Это свойство акустического сигнала может отражать чередование акустической активности, связанной с динамикой сравнительно крупных дислокационных лавин в структуре полосы деформации с периодами ее затухания вследствие релаксации внутренних напряжений, и наоборот, за время относительно низкой активности сигнала АЭ в материале накапливаются внутренние напряжения, последующая релаксация которых дает всплеск акустической активности и т.д.

\section{Заключение}

Проведены in situ исследования динамики формирования полос макролокализованной деформации с помощью синхронной регистрации данных силового отклика, высокоскоростной видеосъемки со скоростью $5000 \mathrm{fps}$ и акустического отклика в полосе частот от $\sim 50 \mathrm{kHz}$ до $\sim 1 \mathrm{MHz}$ в условиях прерывистой ползучести алюминий-магниевого сплава АМг6. Установлено, что самая ранняя и наиболее быстрая стадия формирования полосы деформации, продолжительностью несколько миллисекунд, связанная с зарождением и ростом зародыша полосы, выходом ее на поверхность и последующим быстрым расширением полосы, сопровождается генерированием всплеска АЭ длительностью 3-10 ms. 
Сложная структура этого всплеска включает $\sim 10^{3}$ дискретных импульсов АЭ длительностью $\sim 1-10 \mu \mathrm{s}$ различной амплитуды.

Наиболее информативной характеристикой всплеска АЭ, вызванного формированием одиночной полосы деформации, является форма и амплитуда огибающей акустического всплеска, которые, как установлено, хорошо коррелируют с формой и амплитудой скорости роста скачка разгрузки системы машина-образец.

Выявлены степенные функции распределения амплитуд дискретных сигналов АЭ в структуре акустического всплеска при формировании полосы, а также монофрактальность временной структуры всплеска АЭ с фрактальной размерностью $D_{f} \approx 1.7$, рассчитанной с помощью $R / S$-анализа по методу Хёрста. Полученные результаты свидетельствуют о коллективной самоорганизующейся динамике дислокационных лавин в структуре формирующейся полосы макролокализованной деформации.

Полученные в $[22,23]$ и в настоящей работе результаты дают прямое экспериментальное доказательство дислокационной природы сигналов АЭ, сопровождающих прерывистую деформацию металлов и сплавов, основанное на установленной связи между всплеском сигнала АЭ и динамикой деформационных полос. Кроме того, в этих работах выявлено информационное содержание низкочастотной (в звуковом диапазоне) и высокочастотной (ультразвуковой) составляющей акустического сигнала, генерируемого в ходе формирования деформационных полос в алюминий-магниевом сплаве.

\section{Финансирование работы}

Комплексные высокоскоростные экспериментальные in situ исследования динамики деформационных полос выполнены при частичной поддержке РНФ (проект № 18-19-00304), а статистический и фрактальный анализ акустических сигналов выполнен при поддержке РФФИ (проект № 19-08-00395).

\section{Конфликт интересов}

Авторы заявляют, что у них нет конфликта интересов.

\section{Список литературы}

[1] Панин В.Е., Лихачев В.А., Гриняев Ю.В. Структурные уровни деформации твердых тел. Новосибирск.: Наука, 1985. $230 \mathrm{c}$.

[2] James D.R., Carpenter S.H. // J. App. Phys. 1971. Vol. 42. N 12. P. 4685-4697.

[3] Mathis K., Chmelik F. In: Acoustic Emission, Ed. by Wojciech Sikorski. 2012. P. 23-48.

[4] Бойко В.С., Нацик В.Д. Элементарные процессы пластической деформации кристаллов. Киев.: Наукова думка, 1978. C. $159-189$.

[5] Нацик В.Д., Чишко К.А. // Акустический журнал. 1992. Т. 38. Вып. 3. С. 511-519.
[6] Rouby D., Fleischmann P., Duvergier C. // Phil. Mag. A. 1983. Vol. 47. N 5. P. 671-687.

[7] Rouby D., Fleischmann P., Duvergier C. // Phil. Mag. A. 1983. Vol. 47. N 5. P. 689-705.

[8] Бойко В.С., Гарбер Р.И., Кившик В.Ф. // ФТТ. 1975. Т. 17. Вып. 5. С. 1541-1543.

[9] Hartman W.F. // Exp. Mech. 1974. Vol. 14. P. 19-23. https://doi.org/10.1007/BF02324855.

[10] Криштал М.М., Мерсон Д.Л. // ФММ. 1996. Т. 81. N 1. P. $156-162$.

[11] Chmelik F., Ziegenbein A., Neuhauser H., Lukac P. // Mater. Sci. Eng. A. 2002. Vol. 324. P. 200-207. DOI: $10.1016 / \mathrm{S} 0921-5093(01) 01312-0$

[12] Chmelik F., Klose F.B., Dierke H., Sachl J., Neuhauser H., Lukac P. // Mater. Sci. Eng. A. 2007. Vol. 462. P. 53-60. DOI: https://doi.org/10.1016/j.msea.2006.01.169

[13] Криштал М.М., Хрусталев А.К., Разуваев А.А., Демин И.С. // Деформация и разрушение материалов. 2008. Вып. 1. С. 28-34.

[14] Lebyodkin M.A., Lebedkina T.A., Chmelik F., Lamark T.T., Estrin Y., Fressengeas C., Weiss J. // Phys. Rev. B. 2009. Vol. 79. P. 174114. DOI: https://doi.org/10.1103/PhysRevB.79.174114

[15] Lebyodkin M.A., Kobelev N.P., Bougherira Y., Entemeyer D., Fressengeas C., Gornakov V.S., Lebedkina T.A., Shashkov I.V. // Acta Mater. 2012. Vol. 60. P. 3729-3740. https://doi.org/10.1016/j.actamat.2012.03.029

[16] Shashkov I.V., Lebyodkin M.A., Lebedkina T.A. // Acta Mater. 2012. Vol. 60. P. 6842-6850. https://doi.org/10.1016/j.actamat.2012.08.058

[17] Lebyodkin M.A., Shashkov I.V., Lebedkina T.A. // Phys. Rev. E. 2013. Vol. 88. P. 042402. https://doi.org/10.1103/PhysRevE.88.042402

[18] Kumar J., Sarmah R., Ananthakrishna G. // Phys. Rev. B. 2015. Vol. 92. P. 144109. https://doi.org/10.1103/PhysRevB.92.144109

[19] Криштал М.М., Хрусталев А.К., Волков А.В., Бородин С.А. // ДАН. 2009. Т. 426. Вып. 1. С. 36-40.

[20] Tong W., Tao H., Zhang N., Hector L.G. // Scr. Mater. 2005. Vol. 53. P. 87-92. https://doi.org/10.1016/j.scriptamat.2005.03.020

[21] Xiang G.F., Zhang Q.C., Liu H.W., Wu X.P., Ju X.Y. // Scr. Mater. 2007. Vol. 56. P. 721-724. https://doi.org/10.1016/j.scriptamat.2006.08.049

[22] Шибков А.А., Желтов М.А., Гасанов М.Ф., Золоmoв A.E. // ФТТ. 2017. Т. 59. Вып. 12. С. 23632369. DOI: 10.21883/FTT.2017.12.45232.156. [Shibkov A.A., Zheltov M.A., Gasanov M.F., Zolotov A.E. // Physics Solid State. 2017. Vol. 59. N 12. P. 2387-2393.]

[23] Shibkov A.A., Zheltov M.A., Gasanov M.F., Zolotov A.E., Denisov A.A., Lebyodkin M.A. // Mater. Sci. Eng. A. 2020. Vol. 772. P. 138777. https://doi.org/10.1016/j.msea.2019.138777

[24] Shibkov A.A., Gasanov M.F., Zheltov M.A., Zolotov A.E., Ivolgin V.I. // Int. J. Plast. 2016. Vol. 86. P. 37-55. http://dx.doi.org/10.1016/j.ijplas.2016.07.014

[25] Шибков А.А., Мазилкин А.А., Протасова С.Г., Михлик Д.В., Золотов А.Е., Желтов М.А., Шуклинов А.В. // Деформация и разрушение материалов. 2008. Вып. 5. C. 24-32.

[26] Vinogradov A., Lasarev A. // Scr. Mater. 2012. Vol. 66. P. 745 748. https://doi.org/10.1016/j. scriptamat.2012.01.053 
[27] Jensen H.J. Self-Organized Criticality. Cambridge Univ. Press. Cambridge. 1998. 153 p.

[28] Федер Е. Фракталы. М.: Мир, 1991. 230 с.

[29] Miguel M.C., Vesplignanl A., Zapperi S., Weiss J., Grasso J.R. // Nature. 2001. Vol. 410. N 4. P. 667-671. DOI: $10.1038 / 35070524$

[30] Weiss J., Grasso J.-R. // J. Phys. Chem. B. 1997. Vol. 101. N 32. P. 6113-6117. DOI: 10.1021/jp963157f

[31] Weiss J., Grasso J.-R., Miguel M.-C., Vespignani A., Zapperi S. // Mater. Sci. Eng. A. 2001. Vol. 309-310. P. 360 364. PII: S0921-5093(00)01633-6

[32] Lebyodkin M.A., Kobelev N.P., Bougherira Y., Entemeyer D., Fressengeas C., Gornakov V.S., Lebedkina T.A., Shashkov I.V. // Acta Mater. 2012. Vol. 60. P. 3729-3740. https://doi.org/10.1016/j.actamat.2012.03.026

[33] Lebyodkin M.A., Shashkov I.V., Lebedkina T.A., Mathis K., Dobron P., Chmelik F. // Phys. Rev. E. 2013. Vol. 88. P. 042402. https://doi.org/10.1103/PhysRevE.88.042402

[34] Lebedkina T.A., Zhemchuzhnikova D.A., Lebyodkin M.A. // Phys. Rev. E. 2018. Vol. 97. P. 013001. https://doi.org/10.1103/PhysRevE.97.013001

[35] Shashkov I.V., Lebyodkin M.A., Lebedkina T.A. // Acta Mater. 2012. Vol. 60. P. 6842-6850. https://doi.org/10.1016/j.actamat.2012.08.058

[36] Weiss J., Louchet F. // Scr. Mater. 2006. Vol. 54. P. 747-751. DOI: 10.1016/j.scriptamat.2005.10.056

[37] Weiss J., Richeton T., Louchet F., Chmelik F., Dodron P., Entemeyer D., Lebyodkin M., Lebedkina T., Fressengeas C., McDonald R.J. // Phys. Rev. B. 2007. Vol. 76. P. 224110. https://doi.org/10.1103/PhysRevB.76.224110 